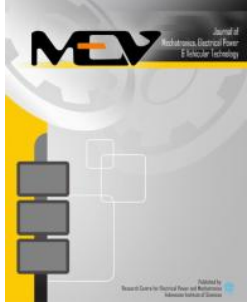

Journal of Mechatronics, Electrical Power, and Vehicular Technology

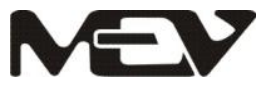

\title{
NONLINEAR DYNAMIC MODELING OF A FIXED-WING UNMANNED Aerial Vehicle: A Case Study of Wulung
}

\author{
Fadjar Rahino Triputra ${ }^{\mathrm{a}, \mathrm{c}, *}$, Bambang Riyanto Trilaksono ${ }^{\mathrm{a}}$, Trio Adiono ${ }^{\mathrm{a}}$, \\ Rianto Adhy Sasongko ${ }^{b}$, Mohamad Dahsyat ${ }^{c}$ \\ ${ }^{a}$ School of Electrical Engineering and Informatics, Institut Teknologi Bandung \\ J1. Ganesha 10, Bandung, Indonesia \\ ${ }^{\mathrm{b}}$ Faculty of Mechanical and Aerospace Engineering, Institut Teknologi Bandung \\ Jl. Ganesha 10, Bandung, Indonesia \\ ${ }^{\mathrm{c}}$ Agency for the Assessment and Application of Technology (BPPT) \\ Komplek Puspiptek Serpong, Tangerang, Indonesia
}

Received 13 February 2015; received in revised form 30 June 2015; accepted 30 June 2015

Published online 30 July 2015

\begin{abstract}
Developing a nonlinear adaptive control system for a fixed-wing unmanned aerial vehicle (UAV) requires a mathematical representation of the system dynamics analytically as a set of differential equations in the form of a strict-feedback systems. This paper presents a method for modeling a nonlinear flight dynamics of the fixed-wing UAV of BPPT Wulung in any conditions of the flight altitude and airspeed for the first step into designing a nonlinear adaptive controller. The model was formed into 10DOF differential equations in the form of strict-feedback systems which separates the terms of elevator, aileron, rudder, and throttle from the model. The model simulation results show the behavior of the flight dynamics of the Wulung UAV and also prove the compliance with the actual flight test results.
\end{abstract}

Keywords: fixed-wing UAV; nonlinear flight dynamics; strict-feedback systems.

\section{INTRODUCTION}

A fixed-wing unmanned aerial vehicle (UAV) has many advantages to fulfill important missions in a wide range of territory. Agency for the Assessment and Application of Technology (BPPT) developed a fixed-wing UAV called Wulung as shown in Figure 1, to provide a solution of the country problem for keeping critical assets in a wide territory such as ocean and forest from disaster, illegal fishing, and illegal logging. This UAV has the proficiency to carry a $20 \mathrm{~kg}$ payload and the flight range of 200 $\mathrm{km}$ from the home base for various missions such as surveillance, aerial photography, search and rescue, and weather modification. However, to meet the needs of concerned missions, an adaptive flight control is needed to drive this fixed-wing UAV to the mission locations autonomously. Hence, a nonlinear dynamic model of Wulung UAV is also needed as part of the flight control design to manage the UAV

\footnotetext{
* Corresponding Author: Tel: +62-8158000730

E-mail: fadjar.rahino@bppt.go.id
}

flying to the mission destinations reliably and safely. Afterwards, some modeling studies of Wulung UAV have been conducted to formulate the flight control design.

Formerly, the flight dynamics modeling of a fixed-wing UAV in the case of BPPT Wulung UAV [1] have been conducted using the linear systems approach [2] in which the analytical model aerodynamic coefficients are calculated using DATCOM software [3]. Flight test data of Wulung UAV has also been obtained to identify a linear model using grey-box method [4] that involves the analytical linear model. However, the flight dynamics model in the form of linear state-space cannot handle the changes in altitude and airspeed because the differential equations of flight dynamics use DATCOM aerodynamic coefficients which only can be applied for a specific altitude and airspeed. These studies led to a conclusion to develop a nonlinear Wulung UAV model in any condition of the flight altitude and airspeed for the first step into designing a nonlinear adaptive controller. Consequently, a 


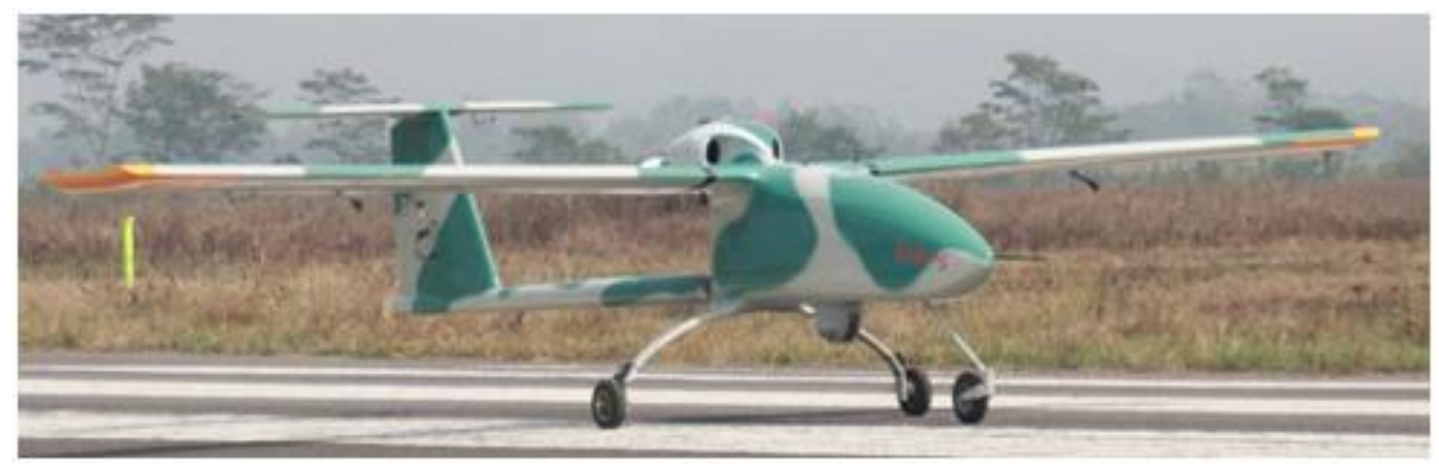

Figure 1. A prototype of BPPT Wulung UAV

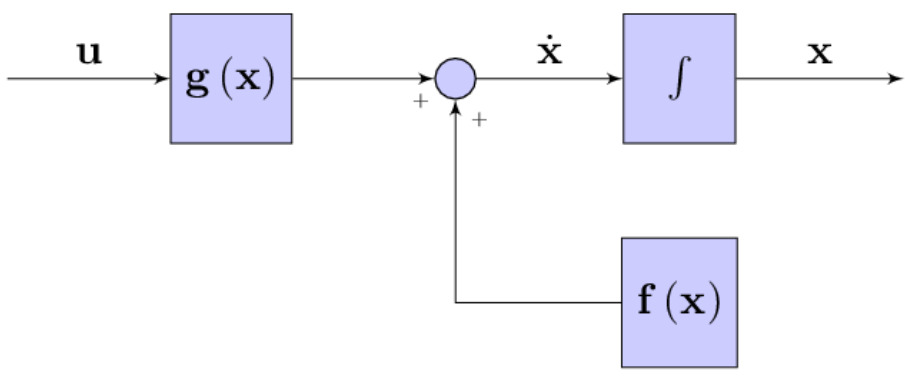

Figure 2. Strict-feedback systems model

practical nonlinear flight dynamic model is required for a chosen adaptive control systems.

Subsequently, an adaptive control systems of an integrator back stepping, that have the advantage to handle the nonlinear model well, have been described by Krstic [5]. Nevertheless, a complicated analytical model derivation must be solved in designing this control systems. Successively, command filtered back stepping (CFBS) has been proposed by Farrell [6][7] that eliminated the requirement of analytical model derivation and simplified its control design. However, introducing the Wulung UAV model to this controller needs a nonlinear model in the form of strict-feedback systems [8][9] that separates the terms of the fixed-wing UAV control variables as shown in Figure 2 and expressed as the following model:

$$
\dot{x}=f(x)+g(x) u
$$

where $\mathrm{x}$ and $\mathrm{u}$ are vectors of state and control variables respectively.

This paper presents the development of a nonlinear flight dynamics model in any conditions of the flight altitude and airspeed by calculating aerodynamic coefficients as functions of altitude and airspeed. The program source of DATCOM software [3] are used as the basis to build analytical equations for aerodynamic coefficients using basic aerodynamics of lifting surfaces [10]. The advantage of this proposed nonlinear model is to eliminate the use of thirdparty software to obtain the aerodynamic coefficients, thus the geometry characteristic of the fixed-wing UAV such as wing span and wing chord can be directly applied. Later, we constructed 10 degrees of freedom (DOF) of differential equations in the form of strictfeedback systems to represent a non-linear dynamic model [2][10][11] using the model of the proposed forces and moments that have input parameters of state variables including the altitude and the airspeed. Simulations of BPPT Wulung UAV in longitudinal and lateral dynamic have been conducted using this model, then their results have been matched with the actual flight test data to prove the compliance of this nonlinear model.

\section{Fixed-Wing UAV Modeling}

Figure 3 shows the movement components of fixed-wing UAV consisting of the attitude $\Xi=\left[\begin{array}{lll}\phi & \theta & \psi\end{array}\right]^{\top}, \quad$ velocity $V=\left[\begin{array}{ll}U & V\end{array}\right]^{\top}, \quad$ angular

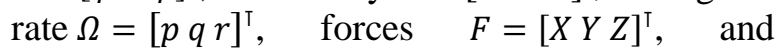
moments $\quad M=\left[L_{\phi} M_{\theta} N_{\psi}\right]^{\top}$ in the vehicle coordinate frame $\left(x_{v}, y_{v}, z_{v}\right)$. Based on the Newton's motion equations of the rigid body, the forces and moments [2][10] are defined as follows:

$$
\begin{aligned}
& F=m \dot{V}+\Omega \times V \\
& M=J \dot{\Omega}+\Omega \times J \Omega
\end{aligned}
$$

where $m$ is the mass and $\mathrm{J}$ is the moment of inertia. 


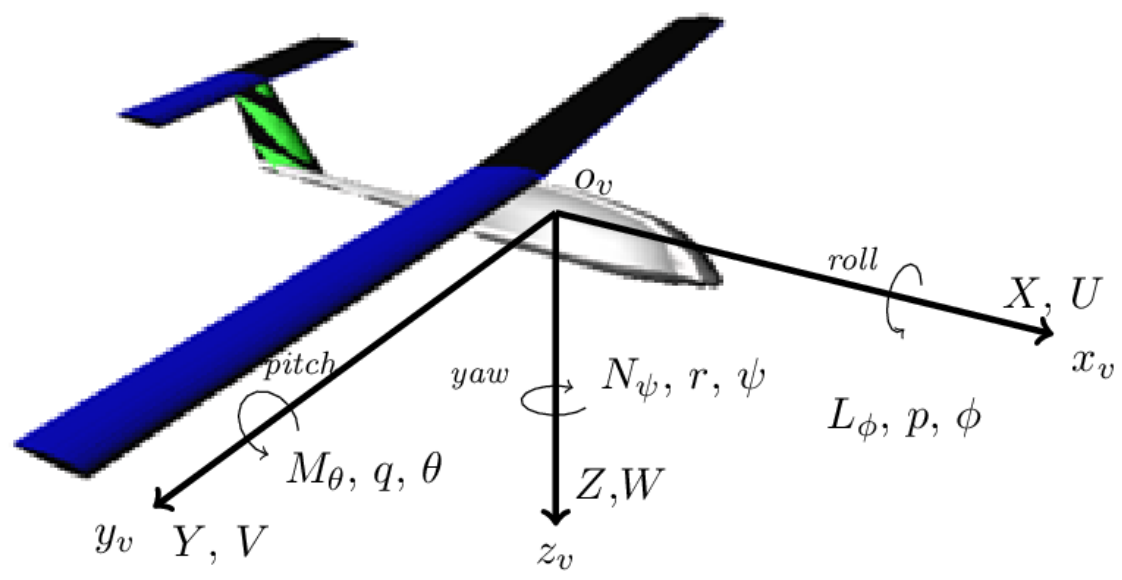

Figure 3. Vehicle coordinate frame components

Hence, the model of fixed-wing UAV is then written in the following equation:

$$
\begin{aligned}
& \dot{V}=\frac{F}{m}-\Omega \times V \\
& \dot{\Omega}=J^{-1}(M-\Omega \times J \Omega)
\end{aligned}
$$

The velocity is generally obtained from inertia sensors such as GPS/INS that provides calculated velocity in an inertia coordinate frame. So these components can be converted into a vehicle coordinate frame as follows:

$$
V={ }^{v} R_{e} V_{\text {gps }}
$$

where $V_{\text {gps }}=\left[V_{\text {nort } h} V_{\text {east }} V_{\text {down }}\right]^{\top}$ is inertia velocity from GPS/INS and ${ }^{v} \mathrm{R}_{e}$ is direct cosine matrix (DCM) as defined as follows:

$$
\begin{aligned}
{ }^{v} R_{e} & ={ }^{v} R_{\phi}{ }^{v} R_{\theta}{ }^{v} R_{\psi} \\
{ }^{v} R_{\phi} & =\left[\begin{array}{ccc}
1 & 0 & 0 \\
0 & \cos \phi & \sin \phi \\
0 & -\sin \phi & \cos \phi
\end{array}\right] \\
{ }^{v} R_{\theta} & =\left[\begin{array}{ccc}
\cos \theta & 0 & -\sin \theta \\
0 & 1 & 0 \\
\sin \theta & 0 & \cos \theta
\end{array}\right] \\
{ }^{v} R_{\psi} & =\left[\begin{array}{ccc}
\cos \psi & \sin \psi & 0 \\
-\sin \psi & \cos \psi & 0 \\
0 & 0 & 1
\end{array}\right]
\end{aligned}
$$

Beside angular rate of equation (5), the calculation of the Euler angular rate $\dot{\Xi}$ that has a link with the following angular rate is required:

$$
\Omega=\left[\begin{array}{c}
\dot{\phi} \\
0 \\
0
\end{array}\right]+{ }^{v} R_{\phi}\left[\begin{array}{l}
0 \\
\dot{\theta} \\
0
\end{array}\right]+{ }^{v} R_{\phi}{ }^{v} R_{\theta}\left[\begin{array}{c}
0 \\
0 \\
\dot{\psi}
\end{array}\right]
$$

Hence, the Euler angular rate is written as follows:

$$
\dot{\Xi}=R_{\Omega} \Omega
$$

where,

$$
R_{\Omega}=\left[\begin{array}{ccc}
1 & \sin \phi \tan \theta & \cos \phi \tan \theta \\
0 & \cos \phi & -\sin \phi \\
0 & \frac{\sin \phi}{\cos \theta} & \frac{\cos \phi}{\cos \theta}
\end{array}\right]
$$

In addition, the altitude rate can be written as follows:

$$
\dot{h}=-V_{\text {down }}=R_{h} V
$$

where,

$$
R_{h}=\left[\begin{array}{lll}
\sin \theta & -\sin \phi \cos \theta & -\cos \phi \cos \theta
\end{array}\right]
$$

Therefore, a nonlinear fixed-wing UAV model is obtained using equations (4), (5), (12), and (14) as 10-DOF of differential equations. Thereafter, the forces and the moments due to the influence of vehicle aerodynamics, propeller thrust, and gravity must be described as follows:

$$
\begin{aligned}
& F=F_{a}+F_{p}+F_{g} \\
& M=M_{a}+M_{p}
\end{aligned}
$$

where $F_{a}$ and $M_{a}$ respectively are aerodynamic forces and moments, $F_{p}$ and $M_{p}$ respectively are propeller thrust forces and moments, and $F_{g}$ is gravity forces.

\section{A. Aerodynamic Forces and Moments}

Figure 4 shows three frames of coordinate, namely the vehicle coordinate frame $\left(x_{v}, y_{v}, z_{v}\right)$, the stability coordinate frame $\left(x_{s}, y_{s}, z_{s}\right)$, and the wind coordinate frame $\left(x_{w}, y_{w}, z_{w}\right)$. Aerodynamic forces and moments, that occurs in fixed-wing UAV, generally are caused by three kind of forces in the wind coordinate frame, i.e. lift force $L$, drag force $D$, and side force $S$. Then the aerodynamic forces in vehicle coordinate frame is written as follows:

$$
F_{a}={ }^{v} R_{S}{ }^{s} R_{w}\left[\begin{array}{lll}
-D & S & -L
\end{array}\right]^{\top}
$$

where,

$$
\begin{aligned}
& { }^{v} R_{s}=\left[\begin{array}{ccc}
\cos \alpha & 0 & -\sin \alpha \\
0 & 1 & 0 \\
\sin \alpha & 0 & \cos \alpha
\end{array}\right] \\
& { }^{s} R_{w}=\left[\begin{array}{ccc}
\cos \beta & \sin \beta & 0 \\
-\sin \beta & \cos \beta & 0 \\
0 & 0 & 1
\end{array}\right]
\end{aligned}
$$




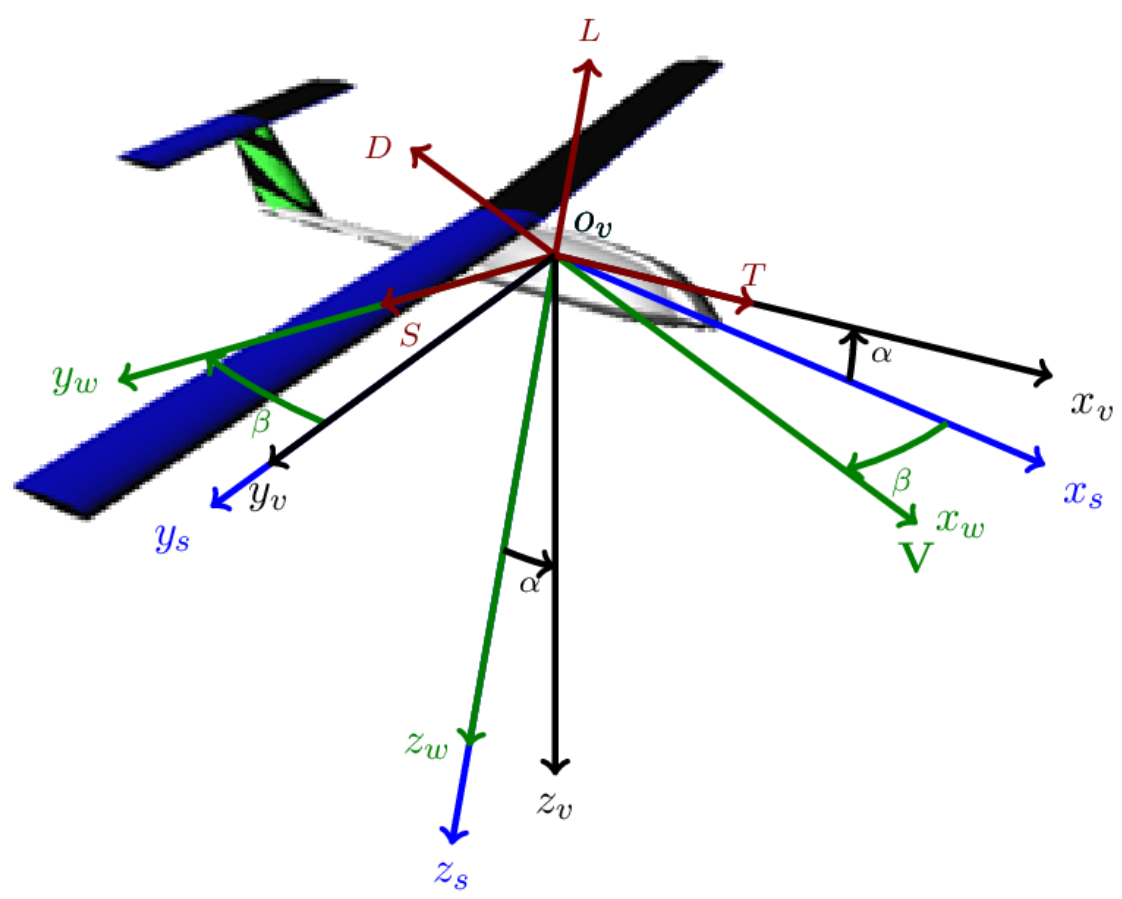

Figure 4. Aerodynamic and thrust forces

Whereas the aerodynamic moments in vehicle coordinate frame is also written as follows:

$$
M_{a}=\left[\begin{array}{lll}
L_{\phi_{a}} & M_{\theta_{a}} & N_{\psi_{a}}
\end{array}\right]^{\top}
$$

In order to form strict-feedback system, the forces and moments must be separated into two kind of forces and moments, i.e. affected by the control surfaces (elevator $\delta_{e}$, aileron $\delta_{a}$, and rudder $\delta_{r}$ ) or not affected. Then equations (18) and (21) are rewritten as follows:

$$
\begin{aligned}
& F_{a}=F_{a_{v}}+C_{F_{a_{\delta}}}\left[\begin{array}{lll}
\delta_{e} & \delta_{a} & \delta_{r}
\end{array}\right]^{\top} \\
& M_{a}=M_{a_{v}}+C_{M_{a_{\delta}}}\left[\begin{array}{lll}
\delta_{e} & \delta_{a} & \delta_{r}
\end{array}\right]^{\top}
\end{aligned}
$$

Hence, the lift, drag, and side forces in wind coordinate frame must also be separated as it is done to aerodynamic forces in vehicle coordinate frame, then equations (22) and (23) are broken down into the following equation:

$$
\begin{aligned}
& F_{a_{v}}={ }^{v} R_{s}{ }^{s} R_{w}\left[\begin{array}{lll}
-D_{v} & S_{v} & -L_{v}
\end{array}\right]^{\top} \\
& C_{F_{a_{\delta}}}={ }^{v} R_{s}{ }^{s} R_{w}\left[\begin{array}{ccc}
-C_{D_{e}} & 0 & -C_{D_{r}} \\
0 & 0 & C_{S_{r}} \\
-C_{L_{e}} & 0 & 0
\end{array}\right] \\
& M_{a_{v}}=\left[\begin{array}{lll}
L_{\phi_{v}} & M_{\theta_{v}} & N_{\psi_{v}}
\end{array}\right]^{\top} \\
& C_{M_{a_{\delta}}}=\left[\begin{array}{ccc}
0 & C_{L_{\phi_{a}}} & C_{L_{\phi_{r}}} \\
C_{M_{\theta_{e}}} & 0 & 0 \\
0 & C_{N_{\psi_{a}}} & C_{N_{\psi_{r}}}
\end{array}\right]
\end{aligned}
$$

Afterwards, the lift, drag, and side forces are calculated as follows:

$$
L_{v}=\frac{1}{2} \rho V_{a}^{2} S_{w}\left(C_{L_{0}}+C_{L_{\alpha}} \alpha\right)
$$

$$
\begin{aligned}
& C_{L_{e}}=\frac{1}{2} \rho V_{a}^{2} S_{w} C_{L_{\delta_{e}}} \\
& S_{v}=\frac{1}{2} \rho V_{a}^{2} S_{w} C_{S_{\beta}} \beta \\
& C_{S_{r}}=\frac{1}{2} \rho V_{a}^{2} S_{w} C_{S_{\delta_{r}}} \\
& D_{v}=\frac{1}{2} \rho V_{a}^{2} S_{w}\left(C_{D_{0}}+C_{D_{\alpha}} \alpha+C_{D_{\beta}} \beta\right) \\
& C_{D_{e}}=\frac{1}{2} \rho V_{a}^{2} S_{w} C_{D_{\delta_{e}}} \\
& C_{D_{r}}=\frac{1}{2} \rho V_{a}^{2} S_{w} C_{D_{\delta_{r}}}
\end{aligned}
$$

In addition, the rolling, pitching, and yawing moments are calculated as follows:

$$
\begin{aligned}
& L_{\phi_{v}}=\frac{1}{2} \rho V_{a}^{2} S_{w} b_{w} C_{L_{\phi_{\beta}}} \beta \\
& C_{L_{\phi_{a}}}=\frac{1}{2} \rho V_{a}^{2} S_{w} b_{w} C_{L_{\phi_{\delta_{a}}}} \\
& C_{L_{\phi_{r}}}=\frac{1}{2} \rho V_{a}^{2} S_{w} b_{w} C_{L_{\phi_{\delta_{r}}}} \\
& M_{\theta_{v}}=\frac{1}{2} \rho V_{a}^{2} S_{w} b_{w}\left(C_{M_{\phi_{0}}}+C_{M_{\phi_{\alpha}}} \alpha\right) \\
& C_{{M_{\theta_{e}}}}=\frac{1}{2} \rho V_{a}^{2} S_{w} b_{w} C_{M_{\theta_{\delta_{e}}}} \\
& N_{\psi_{v}}=\frac{1}{2} \rho V_{a}^{2} S_{w} b_{w} C_{N_{\psi_{\beta}}} \beta \\
& C_{N_{\psi_{a}}}=\frac{1}{2} \rho V_{a}^{2} S_{w} b_{w} C_{N_{\psi_{\delta_{a}}}} \\
& C_{N_{\psi_{r}}}=\frac{1}{2} \rho V_{a}^{2} S_{w} b_{w} C_{N_{\psi_{\delta_{r}}}}
\end{aligned}
$$

where $\rho$ is the air density at altitude $h, V_{a}$ is the true airspeed, $S_{w}$ is the main wing surface area, and $b_{w}$ is the main wingspan. 
In linear system approach, all of those coefficients $C_{*}$ in equations (28) until (42) are assumed as constants. However, those coefficients are also depend on the true airspeed $V_{a}$ and the altitude $h$. Hence, all of the coefficients are calculated into functions of $C_{*}\left(V_{a}, h\right)$. Note that $*$ denotes any character. Subsequently, the aerodynamics coefficients are written as follows:

$$
\begin{aligned}
& C_{L_{0}}=C_{L_{w_{\alpha}}}\left\{\eta_{w}-\alpha_{w_{0}}\right\}+C_{L_{h_{\alpha}}} \frac{q_{h}}{q_{\infty}} \frac{S_{h}}{S_{w}} \\
& \left\{\frac{d \varepsilon}{d \alpha}\left(\alpha_{w_{0}}-\eta_{w}\right)-\alpha_{h_{0}}+\eta_{h}\right\} \\
& C_{L_{\alpha}}=C_{L_{w_{\alpha}}}+C_{L_{h_{\alpha}}} \frac{q_{h}}{q_{\infty}} \frac{S_{h}}{S_{w}}\left\{1-\frac{d \varepsilon}{d \alpha}\right\} \\
& C_{L_{\delta_{e}}}=C_{L_{h_{\delta_{e}}}} \frac{q_{h}}{q_{\infty}} \frac{S_{h}}{S_{w}} \\
& C_{S_{\beta}}=C_{S_{v_{\beta}}} \frac{q_{h}}{q_{\infty}} \frac{S_{v}}{S_{w}} \\
& C_{S_{\delta_{r}}}=C_{S_{v_{\delta_{r}}}} \frac{q_{h}}{q_{\infty}} \frac{S_{v}}{S_{w}} \\
& C_{D_{0}}=C_{D_{w_{p}}}+C_{D_{h_{p}}} \frac{q_{h}}{q_{\infty}} \frac{S_{h}}{S_{w}}+C_{D_{v_{p}}} \frac{q_{h}}{q_{\infty}} \frac{S_{v}}{S_{w}} \\
& +\frac{\left\{C_{L_{w_{\alpha}}}\left(\eta_{w}-\alpha_{w_{0}}\right)\right\}^{2}}{\pi A_{w} e_{w}} \\
& +\frac{\left\{C_{L_{h_{\alpha}}}\left(\alpha_{h}(0)-\alpha_{h_{0}}\right)\right\}^{2}}{\pi A_{h} e_{h}} \frac{q_{h}}{q_{\infty}} \frac{S_{h}}{S_{w}} \\
& +\frac{2 C_{L h_{\alpha}}{ }^{2}\left(\alpha_{h}(0)-\alpha_{h_{0}}\right)}{\pi A_{h} e_{h}} \frac{q_{h}}{q_{\infty}} \frac{S_{h}}{S_{w}} \eta_{h} \\
& C_{D_{\alpha}}=\frac{2 C_{L_{w_{\alpha}}}{ }^{2}\left(\alpha+\eta_{w}-\alpha_{w_{0}}\right)}{\pi A_{w} e_{w}} \\
& =\frac{2 C_{L_{h_{\alpha}}}{ }^{2}\left(\alpha_{h}(\alpha)-\alpha_{h_{0}}\right)}{\pi A_{h} e_{h}} \frac{q_{h}}{q_{\infty}} \frac{S_{h}}{S_{w}} \\
& \left\{1-\frac{d \varepsilon}{d \alpha}\right\} \\
& C_{D_{\beta}}=\frac{2 C_{S_{v_{\beta}}}^{2}\left(\beta-\beta_{v_{0}}\right)}{\pi A_{v} e_{v}} \frac{q_{h}}{q_{\infty}} \frac{S_{v}}{S_{w}} \\
& C_{D_{\delta_{e}}}=\frac{2 C_{L_{h_{\alpha}}}\left(\alpha_{h}(\alpha)-\alpha_{h_{0}}\right)}{\pi A_{h} e_{h}} C_{L_{h_{\delta_{e}}}} \frac{q_{h}}{q_{\infty}} \frac{S_{h}}{S_{w}} \\
& C_{D_{\delta_{r}}}=\frac{2 C_{S_{v_{\beta}}}\left(\beta-\beta_{v_{0}}\right)}{\pi A_{v} e_{v}} C_{S_{v_{\delta_{r}}}} \frac{q_{h}}{q_{\infty}} \frac{S_{v}}{S_{w}} \\
& C_{L_{\phi_{\beta}}}=C_{L_{\phi_{w_{\beta}}}}+C_{L_{\phi_{h}}} \frac{q_{h}}{q_{\infty}} \frac{S_{h}}{S_{w}} \frac{b_{h}}{b_{w}} \\
& +C_{L_{\phi_{v}}} \frac{q_{h}}{q_{\infty}} \frac{S_{v}}{S_{w}} \frac{b_{v}}{b_{w}} \\
& C_{L_{\phi_{\delta_{\alpha}}}}=C_{L_{\phi_{w_{\delta}}}} \\
& C_{L_{\phi_{\delta_{r}}}}=C_{S_{v_{\delta_{r}}}} Z_{\mathrm{MAC}} \frac{q_{h}}{q_{\infty}} \frac{S_{v}}{S_{w} b_{w}}
\end{aligned}
$$

$$
\begin{aligned}
& C_{M_{\phi_{0}}}=C_{M_{\phi_{w_{\mathrm{ac}}}}}+C_{M_{\phi_{f_{0}}}} \\
& +C_{L_{w_{\alpha}}}\left(\eta_{w}-\alpha_{w_{0}}\right) \\
& +C_{L_{w_{\alpha}}}\left(\eta_{w}-\alpha_{w_{0}}\right) \frac{x_{\mathrm{ac}_{w f}}-x_{\mathrm{cg}}}{\bar{c}} \\
& -C_{L_{h_{\alpha}}} \frac{x_{\mathrm{cg}}-x_{\mathrm{ac}}}{\overline{\bar{c}}} \frac{q_{h}}{q_{\infty}} \frac{S_{h}}{S_{w}} \\
& \left\{\frac{d \varepsilon}{d \alpha}\left(\alpha_{w_{0}}-\eta_{w}\right)-\alpha_{h_{0}}\right\} \\
& C_{M_{\phi_{\alpha}}}=C_{L_{w_{\alpha}}} \frac{x_{\mathrm{ac}_{w f}}-x_{\mathrm{cg}}}{\overline{\bar{c}}} \\
& -C_{L_{h_{\alpha}}} \frac{x_{\mathrm{cg}}-x_{\mathrm{ac}_{h}}}{\overline{\bar{c}}} \frac{q_{h}}{q_{\infty}} \frac{S_{h}}{S_{w}} \\
& \left\{1-\frac{d \varepsilon}{d \alpha}\right\} \\
& C_{M_{\theta_{\delta_{e}}}}=-C_{L_{h_{\delta_{e}}}} \frac{x_{\mathrm{cg}}-x_{\mathrm{ac}_{h}}}{\overline{\bar{c}}} \frac{q_{h}}{q_{\infty}} \frac{S_{h}}{S_{w}} \\
& C_{N_{\psi_{\beta}}}=-C_{S_{v_{\beta}}} \frac{x_{\mathrm{cg}}-x_{\mathrm{ac}} v}{\overline{\bar{c}}} \frac{q_{h}}{q_{\infty}} \frac{S_{v}}{S_{w}} \\
& C_{N_{\psi a}}=C_{N_{\psi_{w}}} \\
& C_{N_{\psi_{\delta_{r}}}}=-C_{S_{v_{\delta_{r}}}} \frac{x_{\mathrm{cg}}-x_{\mathrm{ac} v}}{\bar{c}} \frac{q_{h}}{q_{\infty}} \frac{S_{v}}{S_{w}}
\end{aligned}
$$

where $\eta_{w}$ and $\eta_{h}$ respectively are wing and horizontal tail plane (HTP) rigging angles, $A_{w}, A_{h}, A_{v}$ respectively are wing, HTP, and vertical tail plane (VTP) aspect ratios, $\alpha_{w_{0}}, \alpha_{h_{0}}, \quad \beta_{v_{0}}$ respectively are wing, HTP, and VTP angles of attack at zero-lift, $\frac{q_{h}}{q_{\infty}}$ is HTP dynamic pressure ratio, $\frac{d \varepsilon}{d \alpha}$ is downwash gradient, $e_{w}, e_{h}, e_{v}$ respectively are wing, HTP, and VTP Oswald coefficient, $S_{h}$ and $S_{v}$ respectively are HTP and VTP surface area, $b_{h}$ and $b_{v}$ respectively are HTP and VTP span. $\alpha_{h}(\alpha)$ is HTP angle of attack due to the vehicle angle of attack that is formulated as follows:

$$
\alpha_{h}(\alpha)=\alpha+\eta_{h}-\eta_{w}-\frac{d \varepsilon}{d \alpha}\left\{\alpha-\alpha_{w_{0}}\right\}
$$

$\overline{\bar{c}}$ is wing mean aerodynamic chord (MAC) length, $x_{\mathrm{cg}}$ is center position of gravity, $x_{\mathrm{ac}_{w f}}, x_{\mathrm{ac}_{h}}, x_{\mathrm{ac}_{v}}$ respectively are wing-fuselage, HTP, and VTP aerodynamic center (a.c.) positions, and $z_{\mathrm{MAC}_{\mathrm{v}}}$ is VTP MAC normal position. $C_{D_{w_{p}}}, C_{D_{h_{p}}}, C_{D_{v_{p}}}$ respectively are wing, HTP, and VTP parasite drag. $C_{M_{\phi_{w_{a}}}}$ and $C_{M_{\phi_{f}}}$ respectively are wing and fuselage rolling moment coefficient at $\alpha=0$. $C_{N_{\psi_{w_{\delta}}}}$ is wing yawing moment coefficient due to aileron.

Furthermore, the wing, HTP, and VTP lift coefficients respectively are defined as follows [10]: 


$$
\begin{aligned}
& C_{L_{w_{\alpha}}}=\frac{2 \pi A_{w}}{2+\sqrt{\frac{A_{w}\left\{1-\left(\frac{V_{a}}{a}\right)^{2}\right\}}{\left(\frac{{ }^{c} l_{w_{0}}}{2 \pi}\right)^{2}}\left\{1+\frac{\left(\tan \Lambda_{\frac{1}{2}}\right)^{2}}{1-\left(\frac{V_{a}}{a}\right)^{2}}\right\}+4}} \\
& C_{L_{h_{\alpha}}}=\frac{2 \pi A_{h}}{2+\sqrt{\frac{A_{h}\left\{1-\left(\frac{V_{a}}{a}\right)^{2}\right\}}{\left(\frac{{ }^{c} l_{h_{0}}}{2 \pi}\right)^{2}}\left\{1+\frac{\left(\tan \Lambda_{\frac{1}{2}}\right)^{2}}{1-\left(\frac{V_{a}}{a}\right)^{2}}\right\}+4}} \\
& C_{S_{v_{\beta}}}=-\frac{2 \pi A_{v}}{2+\sqrt{\frac{A_{v}\left\{1-\left(\frac{V_{a}}{a}\right)^{2}\right\}}{\left(\frac{{ }^{c} l_{v_{0}}}{2 \pi}\right)^{2}}\left\{1+\frac{\left(\tan \Lambda_{\frac{1}{2}}\right)^{2}}{1-\left(\frac{V_{a}}{a}\right)^{2}}\right\}+4}}
\end{aligned}
$$

where $a$ is the speed of sound at altitude $h, c_{l_{w_{\alpha_{0}}}}$, $c_{l_{h_{0}}}, c_{l_{v_{\alpha_{0}}}}$ respectively are wing, HTP, and VTP airfoil lift coefficients, $\Lambda_{\frac{1}{2}}, \Lambda_{\frac{1}{2}}, \Lambda_{\frac{1}{2}}$ respectively are wing, HTP, and VTP half sweep angles.

In addition, the elevator, aileron, and rudder coefficients respectively are defined as follows [10]:

$$
\begin{aligned}
& C_{L_{h_{\delta_{e}}}}=\frac{{ }^{c}{ }_{h_{\delta_{e_{0}}}}}{\sqrt{1-\left(\frac{V_{a}}{a}\right)^{2}}} \\
& C_{L_{\phi_{w a}}}=\frac{{ }_{l_{\phi_{h}}}}{\sqrt{1-\left(\frac{V_{a}}{a}\right)^{2}}}
\end{aligned}
$$

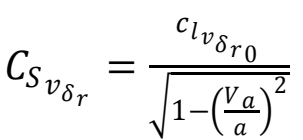

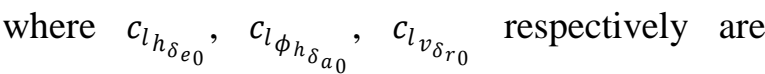
elevator, aileron, and rudder airfoil lift coefficients which are not affected by altitude and airspeed.

The wing, HTP, and VTP rolling moment are defined as follows [10]:

$$
\begin{aligned}
C_{L_{\phi_{w_{\beta}}}=} & -C_{L_{w_{\alpha}}}\left(\eta_{w}-\alpha_{w_{0}}\right) \frac{y_{\mathrm{MAC}}}{b_{w}} \\
& \sin 2 \Lambda_{w_{\mathrm{LE}}}-\Gamma_{w} C_{L_{w_{\alpha}}} \frac{y_{\mathrm{MAC}}}{b_{w}} \\
C_{L_{\phi_{h_{\beta}}}=} & -C_{L_{h_{\alpha}}}\left(\eta_{h}-\alpha_{h_{0}}\right) \frac{y_{\mathrm{MAC}}}{b_{h}} \\
& \sin 2 \Lambda_{h_{\mathrm{LE}}}-\Gamma_{h} C_{L_{h_{\alpha}}} \frac{y_{\mathrm{MAC}}}{b_{h}} \\
C_{L_{\phi_{v_{\beta}}}=} & C_{S_{v_{\beta}}} \frac{z_{\mathrm{MAC}}}{b_{v}}
\end{aligned}
$$

where $y_{\mathrm{MAC}_{w}}$ and $y_{\mathrm{MAC}_{h}}$ respectively are wing and HTP MAC side positions, $\Gamma_{w}$ and $\Gamma_{h}$ respectively are wing and HTP dihedral angles, $\Lambda_{w_{\mathrm{LE}}}$ and $\Lambda_{h_{\mathrm{LE}}}$ respectively are wing and HTP leading-edge sweep angles.

\section{B. Propeller Thrust Forces and Moments}

The relation between the engine power and the thrust generally use the power and thrust coefficients [12][11] that are defined respectively as follows:

$$
\begin{aligned}
& C_{p}(J)=\frac{P}{\rho n_{\text {prop }}^{3} D_{\text {prop }}^{5}} \\
& C_{t}(J)=\frac{T}{\rho n_{\text {prop }}^{2} D_{\text {prop }}^{4}}
\end{aligned}
$$

where $P$ is engine power, $D_{\text {prop }}$ is propeller diameter, $n_{\text {prop }}$ is propeller rotation per second, and $J$ is rate of advance that is also defined as follows [12][11]:

$$
J=\frac{V_{a}}{n_{\text {prop }} D_{\text {prop }}}
$$

Figure 5a and Figure 5b show the power and thrust coefficient for Wulung UAV model that can be represented as polynomial equations as follow:

$$
\begin{aligned}
C_{p}(J)= & a_{C_{p_{1}}} J^{3}+a_{C_{p_{2}}} J^{2}+a_{C_{p_{3}}} \\
& +a_{C_{p_{4}}} \\
C_{t}(J)= & a_{C_{t_{1}}} J^{3}+a_{C_{t_{2}}} J^{2}+a_{C_{t_{3}} J} \\
& +a_{C_{t_{4}}}
\end{aligned}
$$

Then, the relation between the throttle and engine power is proposed as follows:

$$
P\left(\delta_{t}\right)=a_{P_{1}} \delta_{t}^{3}+a_{P_{2}} \delta_{t}^{2}+a_{P_{3}} \delta_{t}+a_{P_{4}}(77)
$$

Figure $5 c$ shows the relation of equation (77) for Wulung UAV. Therefore, a solution for the following equation must be performed to obtain propeller rotation $n$.

$$
P\left(\delta_{t}\right)=a_{n_{1}} n^{3}+a_{n_{2}} n^{2}+a_{n_{3}} n+a_{n_{4}}(78)
$$

where $a_{n_{1}}=-a_{C_{p_{4}}} \rho D_{\text {prop }}^{3}, a_{n_{2}}=-a_{C_{p_{3}}} \rho V_{a} D_{\text {prop }}^{2}$, $a_{n_{3}}=-a_{C_{p_{2}}} \rho V_{a}^{2} D_{\text {prop }}$, and $a_{n_{4}}=-a_{C_{p_{1}}} \rho V_{a}^{3}$.

Hence, the propeller thrust can be calculated as follows:

$$
T=C_{t}\left(\frac{V_{a}}{n_{\text {prop }} D_{\text {prop }}}\right) \rho n^{2} D_{\text {prop }}^{4}
$$

Next, the thrust as shown in Figure $5 \mathrm{~d}$ is linearized as follows:

$$
T=T_{0}+C_{T_{\delta_{t}}} \delta_{t}
$$


Similar to aerodynamic coefficients calculation, $T_{0}$ and $C_{T_{\delta_{t}}}$ are depend on the true airspeed $V_{a}$ and the altitude $h$. Hence, the forces and moments caused by the thrust in vehicle coordinate frame is written as follows:

$$
\begin{aligned}
& F_{p}=F_{p_{v}}+C_{F_{p_{\delta}}} \delta_{t} \\
& M_{p}=M_{p_{v}}+C_{M_{p_{\delta}}} \delta_{t}
\end{aligned}
$$

Similar to aerodynamic forces and moment, the equations (81) and (82) is then broken down as follows:

$$
F_{p_{v}}=\left[\begin{array}{lll}
T_{0} \cos \tau & 0 & -T_{0} \sin \tau
\end{array}\right]^{\top}
$$

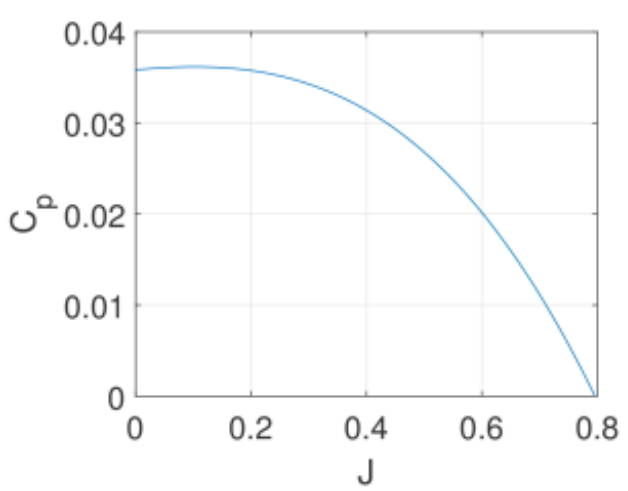

(a)

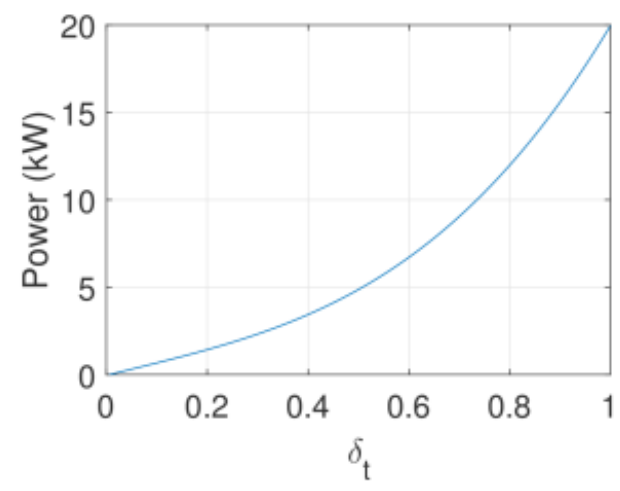

(c)

$$
\begin{aligned}
C_{F_{p_{\delta}}} & =\left[\begin{array}{lll}
C_{T_{\delta_{t}}} \cos \tau & 0 & -C_{T_{\delta_{t}}} \sin \tau
\end{array}\right]^{\top} \\
M_{p_{v}} & =F_{p_{v}} \times P_{\text {prop }} \\
C_{M_{p_{\delta}}} & =C_{F_{p_{\delta}}} \times P_{\text {prop }}
\end{aligned}
$$

where $P_{\text {prop }}=\left[\begin{array}{lll}x_{\text {prop }} & 0 & z_{\text {prop }}\end{array}\right]^{\top}$ is the position of the propeller in vehicle coordinate frame and $\tau$ is propeller rigging angle.

\section{Gravitational Forces}

Figure $6 \mathrm{~b}$ shows the forces of gravitation in inertia coordinate frame, while Figure 6a shows the forces in vehicle coordinate frame. Thus, we write the gravitational forces in vehicle coordinate frame as follows:

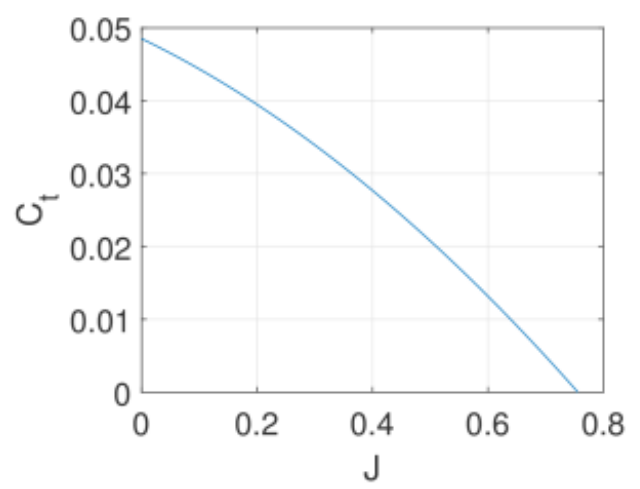

(b)

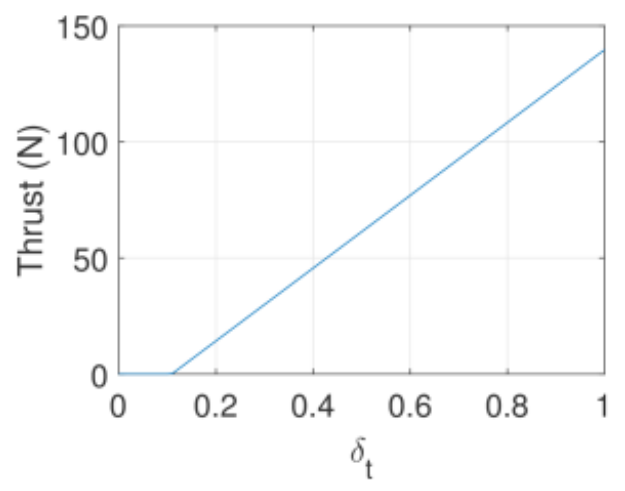

(d)

Figure 5. Wulung propeller model: (a) Power coefficient; (b) Thrust coefficient; (c) Power vs throttle; (d) Thrust vs throttle

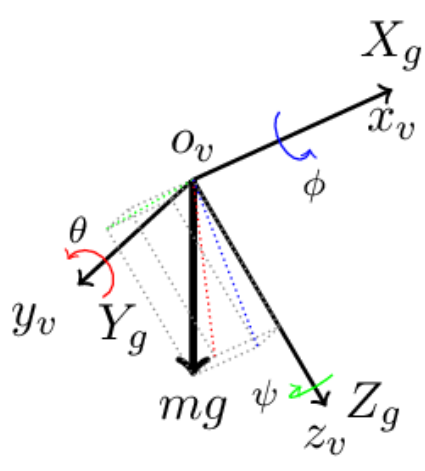

(a)

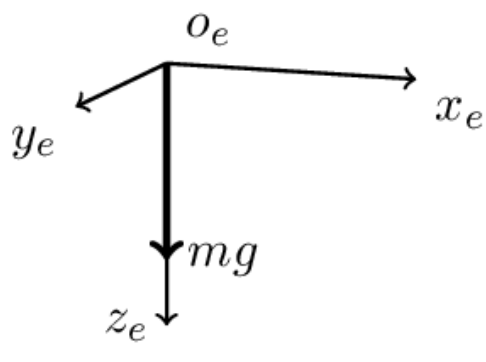

(b)

Figure 6. Gravitational forces: (a) Vehicle frame; (b) Inertia frame 


$$
\begin{aligned}
F_{g} & ={ }^{v} R_{e}\left[\begin{array}{lll}
0 & 0 & m g
\end{array}\right]^{\top} \\
& =m g\left[\begin{array}{lll}
-\sin \theta & \cos \theta \sin \phi & \cos \theta \cos \phi
\end{array}\right]^{\top}(87)
\end{aligned}
$$

where $m$ is mass of the fixed-wing UAV and $g$ is gravitational constant.

\section{Nonlinear Flight Dynamic Model}

The control input $u$ and the state variable $x$ are defined as follows:

$$
\begin{aligned}
& u=\left[\begin{array}{llll}
\delta_{e} & \delta_{a} & \delta_{r} & \delta_{t}
\end{array}\right]^{\top} \\
& x=\left[\begin{array}{llll}
V^{\top} & \Omega^{\top} & \Xi^{\top} & h
\end{array}\right]^{\top}
\end{aligned}
$$

Hence, from equations (12), (14), (24)-(27), (83)-(87), the nonlinear functions $\mathrm{f}(\mathrm{x})$ and $\mathrm{g}(\mathrm{x})$ of equation (1) can be written as follows:

$$
\begin{gathered}
f(x)=\left[\begin{array}{c}
\frac{F_{a_{v}+F_{p_{v}}+F_{g}}}{m}-\Omega \times V \\
J^{-1}\left\{M_{a_{v}}+M_{p_{v}}-\Omega \times J \Omega\right\} \\
R_{\Omega} \Omega \\
R_{h} V
\end{array}\right] \\
g(x)=\left[\begin{array}{cc}
\frac{C_{F a_{\delta}}}{m} & \frac{C_{F p_{\delta}}}{m} \\
J^{-1} C_{M_{a_{\delta}}} & J^{-1} C_{M_{p_{\delta}}} \\
0_{4 \times 3} & 0_{4 \times 1}
\end{array}\right]
\end{gathered}
$$

\section{FLIGHT DYNAMICS RESPONSES}

Wulung UAV profiles in Table 1 are used as model parameters for simulating the responses of the flight dynamics. Two scenarios of disturbance are done by providing doublet angles of elevator and aileron to show the longitudinal and lateral dynamics responses of Wulung UAV.

Before performing simulation, the steady state of flight must be found in the condition that the UAV cruises without changing the altitude by trimming the elevator and throttle. Therefore, if the altitude, throttle, and elevator are respectively set to $h=3000$ feet, $\delta_{t}=67 \%$, and $\delta_{e}=$ $-3.3 \mathrm{deg}$, the steady state condition of Wulung UAV model will be occurred at pitching angle $\theta=1.87 \mathrm{deg}$, axial velocity $U=58.75$ knots, and normal velocity $W=1.21$ knots. Wulung UAV model is then simulated using 10-DOF differential equation (90) and (91). After the calculation, the velocity, angular rate, attitude, and altitude of Wulung UAV are obtained for each simulation scenario.

\begin{tabular}{|c|c|}
\hline Parameter & Unit \\
\hline Mass, $m$ & $120(\mathrm{~kg})$ \\
\hline Wing area, $S_{w}$ & $3.9718\left(\mathrm{~m}^{2}\right)$ \\
\hline Wingspan, $b_{w}$ & $6.355(\mathrm{~m})$ \\
\hline HTP area, $S_{h}$ & $0.819\left(\mathrm{~m}^{2}\right)$ \\
\hline HTP span, $b_{h}$ & $1.95(\mathrm{~m})$ \\
\hline VTP area, $S_{v}$ & $0.519\left(\mathrm{~m}^{2}\right)$ \\
\hline VTP $\operatorname{span}, b_{v}$ & $0.845(\mathrm{~m})$ \\
\hline Wing rigging angle, $\eta_{w}$ & $6^{\circ}$ \\
\hline HTP rigging angle, $\eta_{h}$ & $-3^{\circ}$ \\
\hline Wing dihedral, $\Gamma_{w}$ & $3^{\circ}$ \\
\hline c.g. position, $x_{\mathrm{cg}}$ & $-1.576(\mathrm{~m})$ \\
\hline Wing-fuselage a.c. position, $x_{\mathrm{ac}_{w f}}$ & $-1.497(\mathrm{~m})$ \\
\hline HTP a.c. position, $x_{\mathrm{ac}_{h}}$ & $-4.136(\mathrm{~m})$ \\
\hline VTP a.c. position, $x_{\mathrm{ac}_{v}}$ & $-3.97(\mathrm{~m})$ \\
\hline Wing MAC side position, $y_{\mathrm{MAC}_{w}}$ & $1.589(\mathrm{~m})$ \\
\hline HTP MAC side position, $y_{\mathrm{MAC}_{h}}$ & $0.488(\mathrm{~m})$ \\
\hline VTP MAC normal position, $z_{\mathrm{MAC}_{\mathrm{v}}}$ & $0.294(\mathrm{~m})$ \\
\hline$J_{\mathrm{xx}}$ & $79.045\left(\mathrm{kgm}^{2}\right)$ \\
\hline$J_{\mathrm{yy}}$ & $103.473\left(\mathrm{kgm}^{2}\right)$ \\
\hline$J_{z z}$ & $159.541\left(\mathrm{kgm}^{2}\right)$ \\
\hline$J_{x z}$ & $19.131\left(\mathrm{kgm}^{2}\right)$ \\
\hline Engine max power & 20 (HP) \\
\hline Propeller diameter, $D_{\text {prop }}$ & 32 (inch) \\
\hline
\end{tabular}

\section{A. Longitudinal Dynamics Responses}

In this simulation scenario, the UAV moves westward with the throttle, altitude, velocity, and

Table 1.

Wulung UAV profiles 
attitude as well as steady state condition. Therefore, the elevator angle is set to $\delta_{e}=$ $6.7 \mathrm{deg}$ at time $t=10 \mathrm{sec}, \delta_{e}=-13.3 \mathrm{deg}$ at time $t=10.5 \mathrm{sec}$, and $\delta_{e}=-3.3 \mathrm{deg}$ at time $t=11 \mathrm{sec}$ as shown in Figure 7a.

Figure $7 \mathrm{a}$ also shows the responses of the pitch and attack angles. Once elevator trailing edge down (positive), decreasing the both angles of pitch and attack, and after elevator trailing edge up (negative), increasing the both angles of pitch and attack. Thereafter, the angle of attack immediately return to normal angle less than 3 second, but the pitching angle takes time of damped oscillation to back to steady states. In addition, Figure $7 \mathrm{~b}$ shows the responses of the velocity and altitude. It seems clear that the Wulung UAV suffered Phugoid motion with 2 minutes of damping. This simulation denotes to a conclusion that the longitudinal dynamics characteristic of Wulung UAV is stable without a hard control effort that indicated by the convergence of states.

\section{B. Lateral Dynamics Responses}

Similar to longitudinal simulation scenario, the fixed-wing UAV moves westward in steady

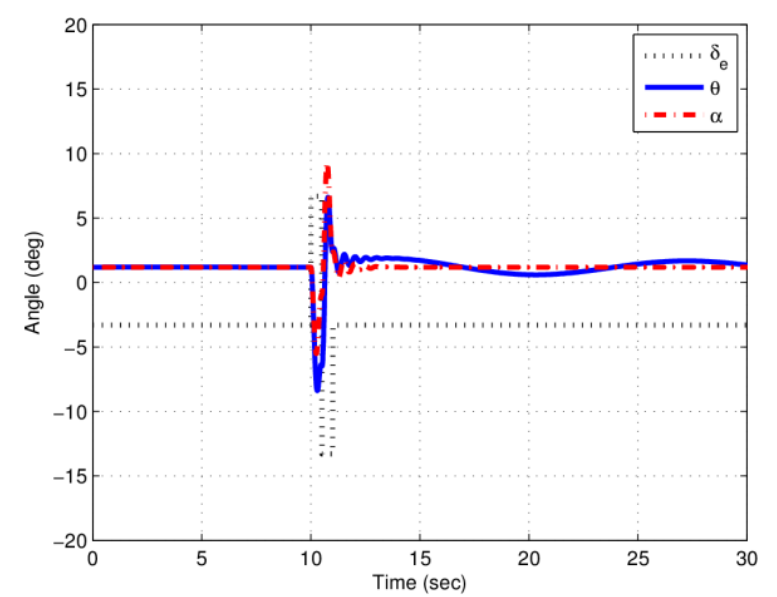

(a)

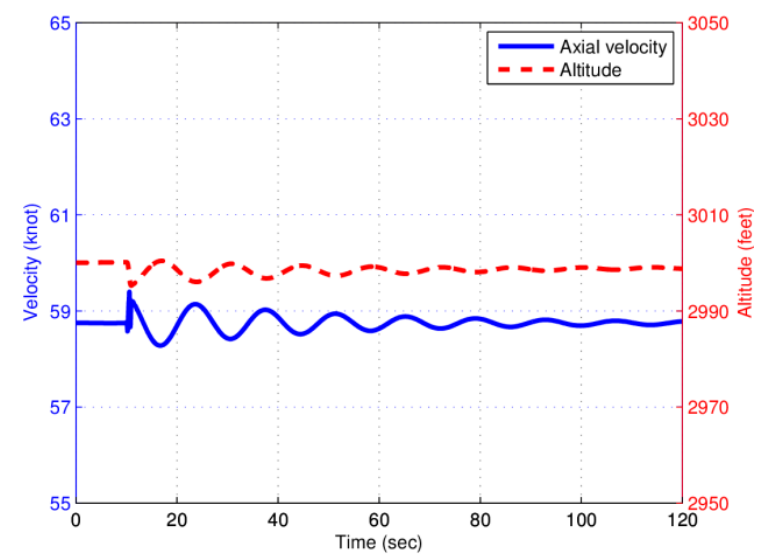

(b)

Figure 7. Wulung longitudinal dynamics responses: (a) Elevator doublet and the responses; (b) The velocity and altitude state condition. Therefore, the aileron angle is set to $\delta_{a}=5 \mathrm{deg}$ at time $t=10 \mathrm{sec}, \delta_{a}=-5 \mathrm{deg}$ at time $t=10.5 \mathrm{sec}$, and aileron angle back to zero again at time $t=11 \mathrm{sec}$ as shown in Figure 8a.

Figure 8a also shows the responses of the roll, pitch, and sideslip angles. Once left aileron trailing edge down (positive), increasing bank angle (roll to right) at high roll rate but decreasing the sideslip angle slightly, and after aileron trailing edge up (negative), still increasing the bank angle at weakened roll rate and also increasing the sideslip angle. Thereafter, the sideslip angle takes time about 2 minutes of damped oscillation to back to the steady state. In contrast, the bank angle decreases to minimum negative angle (roll to left) about 30 seconds and then slowly rises gradually towards steady state in a long time. The aileron doublet disturbance is also reacting on the pitching angle that oscillates for about 3 minutes damping. Figure $8 \mathrm{~b}$ shows the responses of the heading and altitude. It seems clear that Wulung UAV bank angle did not immediately return to the steady state in a long time after aileron doublet disturbance, thus causing the vehicle has a tendency to turn. This

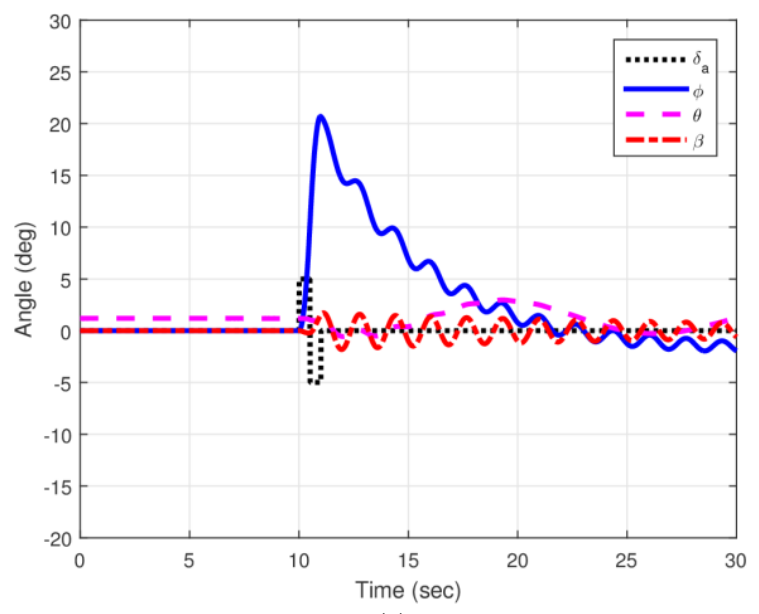

(a)

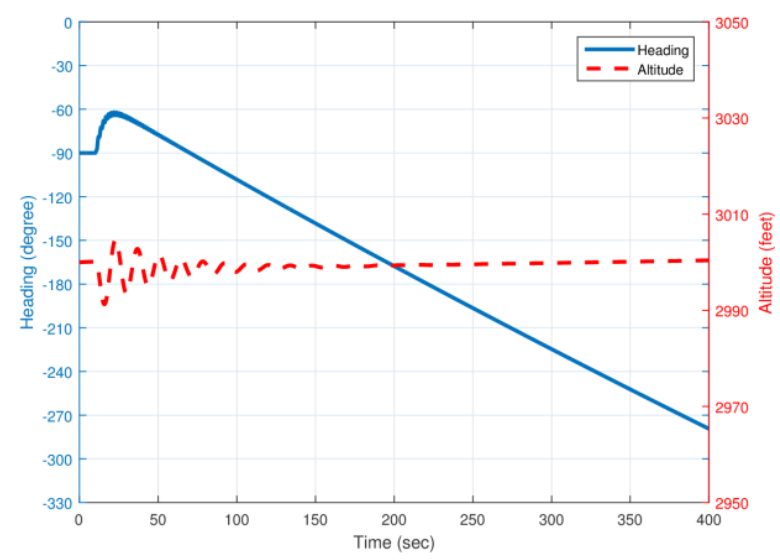

(b)

Figure 8. Wulung lateral dynamics responses: (a) Aileron doublet and the responses; (b) The heading and altitude 
simulation denotes to a conclusion that the lateral dynamics characteristic of Wulung UAV is stable but still need a lateral control to restore the bank angle to the steady state immediately. Wulung UAV also suffered phugoid motion for about 2 minutes that is indicated by pitching angle damped oscillation.

\section{Simulation and Flight TeSt}

A flight test of Wulung UAV has been conducted and the results are compared with model simulation. The elevator doublet of flight test as shown in Figure $9 \mathrm{~b}$ is mimicked into model simulation of longitudinal dynamic as shown in Figure 9a. The difference of physical angles may be caused by initial steady state and the windy conditions when the flight test conducted. Further, both results are normalized so the steady state is same and compared as shown in Figure 9c and Figure 9d.

Figure $9 \mathrm{c}$ shows that both responses of the pitching angle and the angle of attack between model simulation and flight test approached similarity. While Figure 9d shows that pitch rate responses are nearly similar between model simulation and the flight test. Hence, these results demonstrate conformity of model simulation with the actual flight of longitudinal dynamic. The model is better than the system identification method using grey-box [1] because it is compliance with any conditions of altitude and airspeed. Figure 10 shows the response of normalized altitude and velocity so the initial steady state is same. The difference between the simulation and the flight test may be caused by the windy conditions when the flight test conducted.

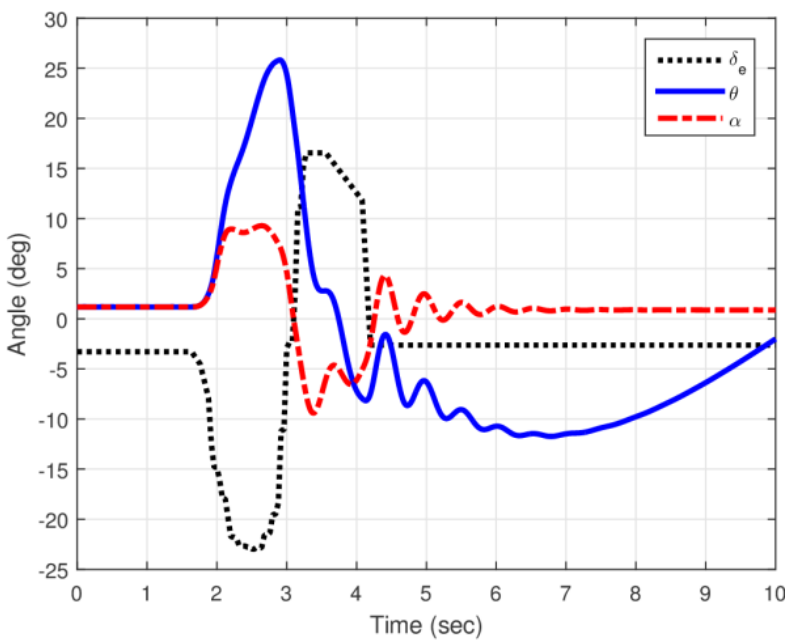

(a)

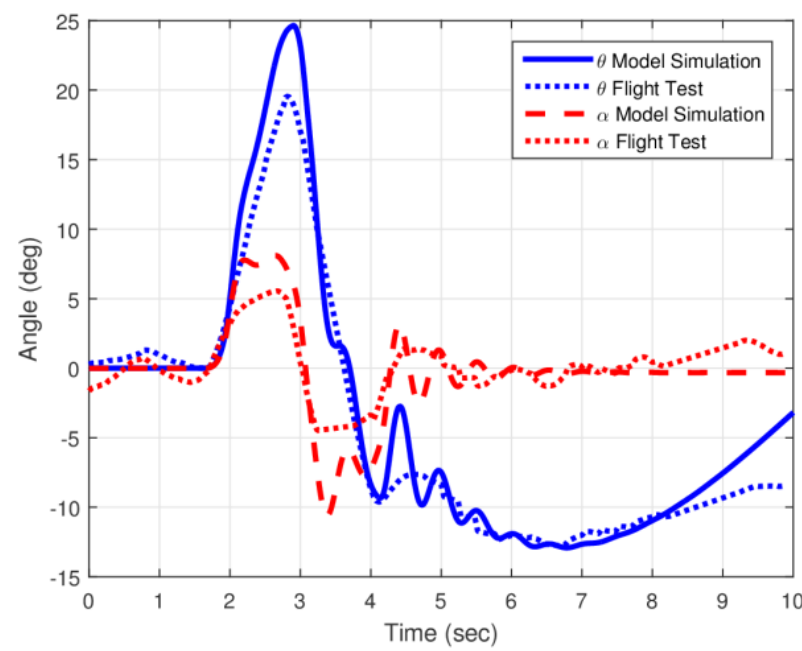

(c)

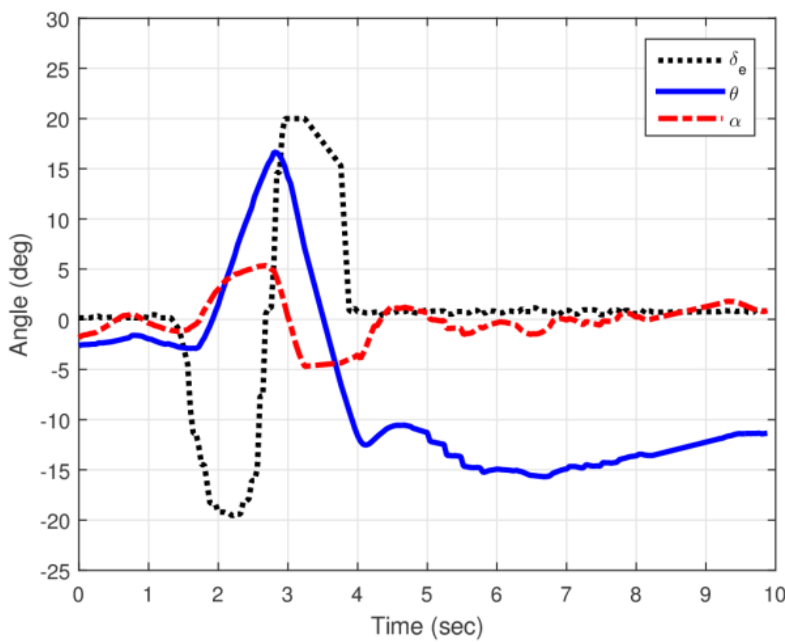

(b)

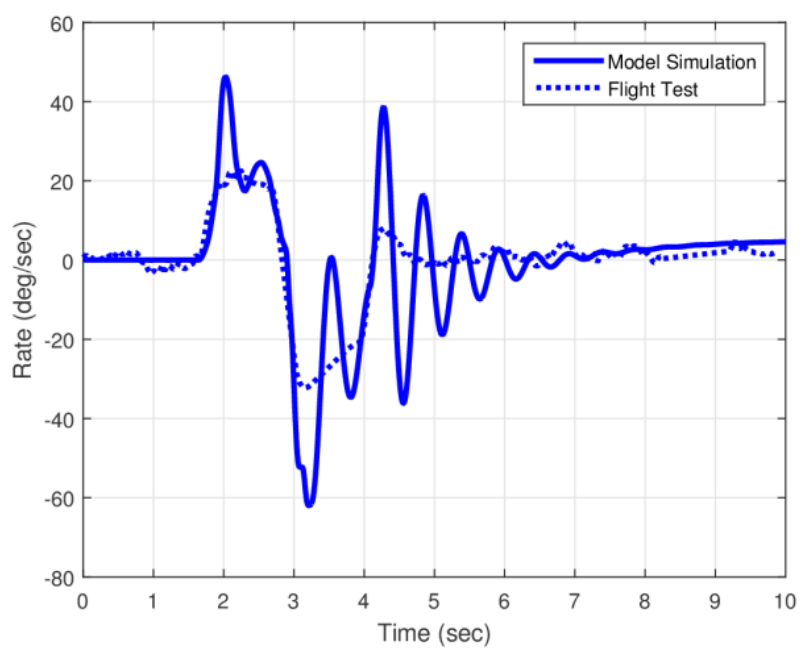

(d)

Figure 9. Model simulation and flight test results of longitudinal dynamics responses; (a) Model simulation results; (b) Flight test results; (c) Pitching and attack angles responses; (d) Pitch rate responses 


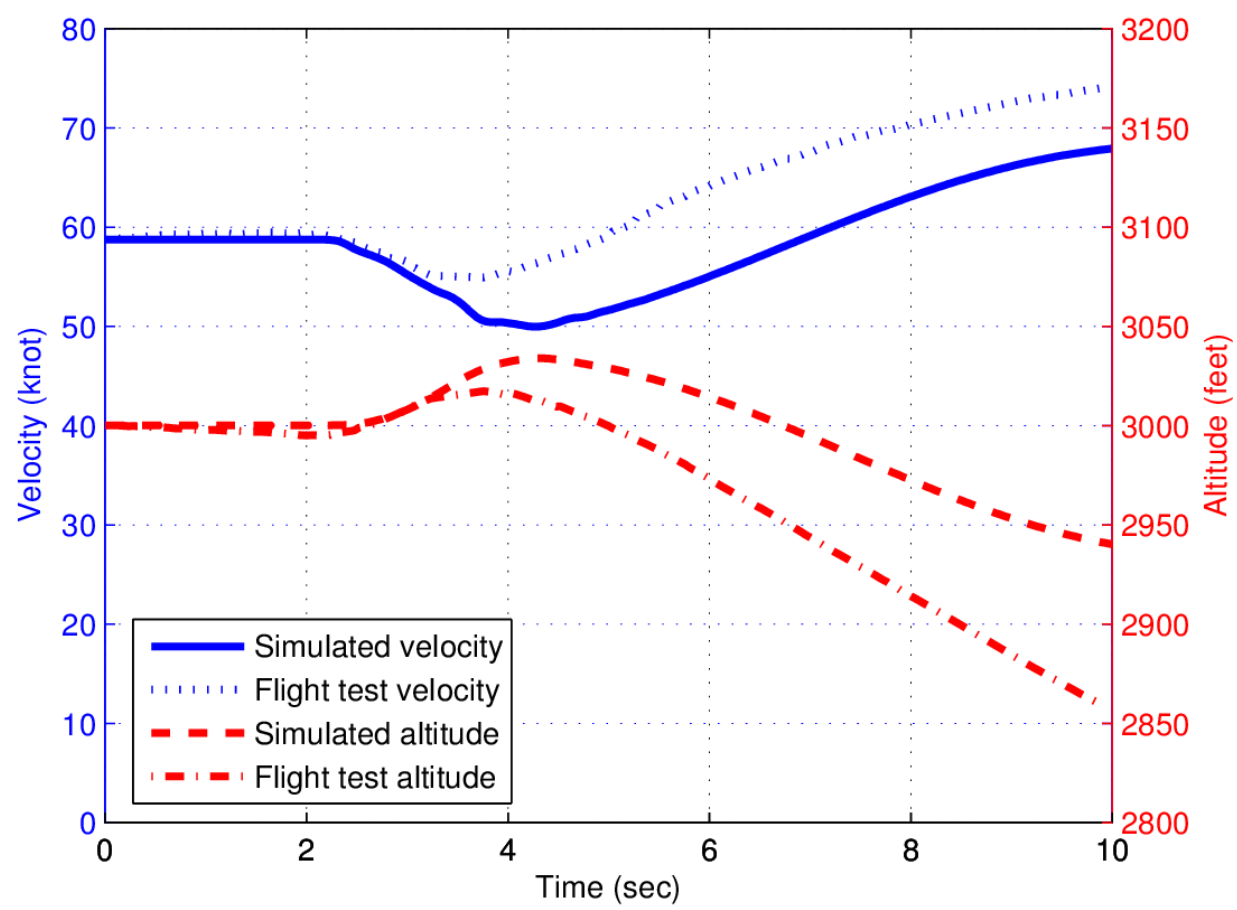

Figure 10. Altitude and velocity responses

\section{CONCLUSION}

The longitudinal and lateral dynamics responses of Wulung UAV are good enough concerning to the outcome of model simulations that show the stability of flying without the hard control effort. Our proposed model is also compliance with flight test results of Wulung UAV. Furthermore, our nonlinear model of the fixed-wing UAV has the advantage to calculate flight dynamics for all conditions of altitude and airspeed that is important to build a controller that adapt to the UAV altitude and airspeed. As future works, this model will be used for an adaptive nonlinear controller using command filtered backstepping method. This model also will be employed for building the hardware in the loop simulation (HILS) systems that very useful for testing the hardware controller module before being used in the field.

\section{ACKNOWLEDGEMENT}

The authors express thanks to UAV team of BPPT Technology Center for Defense and Security Industry, for the support and the providing of required facilities and conducive conditions for this research.

\section{REFERENCES}

[1] F. R. Triputra, et al., "Longitudinal Dynamic System Modeling of a Fixed-Wing UAV towards Autonomous Flight Control System Development: A Case Study of BPPT
Wulung UAV Platform," in Proc. IEEEICSET, Sep. 2012.

[2] M. V. Cook, Flight Dynamics Principles: A Linear Systems Approach to Aircraft Stability and Control. 2nd ed., Massachusetts: Elseivier, 2007.

[3] R. D. Finck, USAF Stability and Control DATCOM. McDonnell Douglas Corp, Wright-Patterson AFB, Ohio,Final Report AFWAL-TR-83-3048, revised, April, 1978.

[4] L. Ljung, System Identification Toolbox Users's Guide for Use with MATLAB, The Math Works Inc., 1995.

[5] M. Krstic, et al., Nonlinear and Adaptive Control Design. New York: John Wiley \& Sons, Inc., 1995, pp. 87-121.

[6] J. A. Farrell and M. M. Polycarpou, Adaptive Approximation Based Control. New Jersey: John Wiley \& Sons, Inc., 2006.

[7] J. A. Farrell, et al., "Command Filtered Backstepping", IEEE Transaction on Automatic Control, 54(6), pp. 1391-1395, June, 2009.

[8] O. Harkegard and S. T. Glad, Flight Control Design Using Backstepping. Linkoping University, Sweden, Rep. No. LiTH-ISY-R2323, 2001.

[9] T. Espinoza, et al., "Backstepping - sliding mode controllers applied to a fixed-wing UAV," in Proc. IEEE-CERMA, May, 2013.

[10] D. K. Schmidt, Modern Flight Dynamics. int. ed., New York: McGraw-Hill, 2012, pp. 156-322. 
[11] R. W. Beard and T. W. McLain, Small Unmanned Aircraft: Theory and Practice. United Kingdom: Princeton University Press, 2012, pp. 8-38.
[12] A. Fillipone, "Propeller Characteristics", 2.4.9, Aerospace Engineering Desk Reference. First ed., San Diego, CA: Elsevier Inc., 2009. 